\title{
A comparison of latanoprost and dorzolamide in patients with glaucoma and ocular hypertension: a 3 month, randomised study
}

\author{
E P O’Donoghue and the UK and Ireland Latanoprost Study Group ${ }^{\star}$
}

\begin{abstract}
Aims-To compare the effects on intraocular pressure (IOP) and side effects of monotherapy with either latanoprost or dorzolamide in patients with glaucoma or ocular hypertension.

Methods-224 patients with open angle glaucoma or ocular hypertension were recruited to a 3 month open labelled study. Previous glaucoma medications were washed out and the patients were randomised to receive either latanoprost $0.005 \%$ once daily or dorzolamide $2 \%$ three times daily.

Results-Of 224 patients 213 were included in the analysis of efficacy. After 3 months, latanoprost reduced mean baseline diurnal IOP from 27.2 (SD 3.0 ) $\mathrm{mm}$ $\mathrm{Hg}$ by 8.5 (3.3) $\mathrm{mm} \mathrm{Hg}$. The corresponding figures for dorzolamide were 27.2 (3.4) and $5.6(2.6) \mathrm{mm} \mathrm{Hg}$. The difference of 2.9 mm Hg (95\% CI: 2.3-3.6) was highly significant $(p<0.001$, ANCOVA). Latanoprost reduced IOP at peak by $8.6 \mathrm{~mm} \mathrm{Hg}$ (32\%) compared with $6.2 \mathrm{~mm} \mathrm{Hg}(23 \%)$ for dorzolamide, and the difference of $2.4 \mathrm{~mm} \mathrm{Hg}$ was significant $(\mathrm{p}<0.001$, ANCOVA). The corresponding figures at trough were $8.1 \mathrm{~mm} \mathrm{Hg}(31 \%)$ for latanoprost and $4.7 \mathrm{~mm} \mathrm{Hg}(17 \%)$ for dorzolamide, a significant difference of $3.4 \mathrm{~mm}$ Hg ( $p<0.001$, ANCOVA). Both drugs were well tolerated systemically and locally. Conclusion-Latanoprost was superior to dorzolamide in reducing the IOP, judged both from the effect on IOP at peak and trough and by the effect on diurnal IOP. (Br f Ophthalmol 2000;84:579-582)
\end{abstract}

The advent of several new drugs for treatment of open angle glaucoma necessitates controlled clinical trials to assess relative efficacy and safety. One of these new agents is dorzolamide, the first topical carbonic anhydrase inhibitor. When dorzolamide $2 \%$ is applied three times daily, it causes a maintained reduction of the intraocular pressure $(\mathrm{IOP})^{1}$ due to reduced aqueous flow. ${ }^{2}$ Another new treatment is the prostaglandin analogue latanoprost which is a potent ocular hypotensive agent. Latanoprost $0.005 \%$ applied once daily reduces IOP at least as effectively as timolol $0.5 \%$ applied twice daily. ${ }^{3-5}$ The effect of latanoprost is induced by increased uveoscleral outflow. ${ }^{6}$ Both dorzolamide and latanoprost are now widely used in the treatment of open angle glaucoma. They are effective pressure lowering agents and appear to have acceptable safety profiles. The aim of the present study was to compare the efficacy and side effects of the two drugs used as single agents.

\section{Patients and methods}

The study was designed as a 3 month, randomised, parallel group, open label, multicentre clinical trial comparing the efficacy and side effects of monotherapy with either latanoprost and dorzolamide. Twelve centres participated and a total of 224 patients were recruited. Approvals were obtained from the appropriate local regulatory authorities and ethics committees for each centre and a signed informed consent was obtained from all patients before study enrolment. The study was performed according to the Declaration of Helsinki.

Patients, 18 years of age or older, with unilateral or bilateral primary open angle glaucoma, capsular glaucoma, or ocular hypertension with an IOP of at least $21 \mathrm{~mm} \mathrm{Hg}$ on previous treatment or $25 \mathrm{~mm} \mathrm{Hg}$ without treatment were eligible for inclusion.

Exclusion criteria included previous treatment with carbonic anhydrase inhibitors or latanoprost, closed or barely open anterior chamber angle or a history of angle closure, current use of contact lenses, intraocular surgery within the past 6 months, argon laser trabeculoplasty within the past 3 months, any ocular inflammation or infection within the past 3 months, known hypersensitivity to any component of the study drugs, or any condition preventing reliable applanation tonometry. Also, patients who had participated in a clinical trial within the past month, were pregnant or nursing, or considering pregnancy, or were unable to adhere to the protocol were also excluded.

If both eyes were eligible they were both accepted as study eyes and the mean value of the IOP in the two eyes was used in the analysis.

The schedule of examinations is presented in Table 1. A prestudy visit was scheduled 2-4 weeks before the baseline examination. During the prestudy visit a medical and ocular history was taken and any concomitant medications were recorded. An eye examination was performed including determination of visual acuity, refraction, a slit lamp examination, ophthalmoscopy, automated perimetry, and IOP measurement. Before treatment with the study drugs any previous glaucoma drugs were washed out. The minimum washout periods were 5 days for cholinergic agonists, 1 week for adrenergic agonists, and 3 weeks for adrenergic
Accepted for publication 27 January 2000 
Table 1 Schedule of examinations and procedures

\begin{tabular}{llllll}
\hline Examinations & Prestudy & Baseline & Week 2 & Month 3 & Follow up \\
\hline Medical and ocular history & $\mathrm{X}$ & & & \\
Visual acuity & $\mathrm{X}$ & $\mathrm{X}$ & $\mathrm{X}$ & $\mathrm{X}$ & \\
Refraction & $\mathrm{X}$ & $\mathrm{X})$ & $(\mathrm{X})$ & $(\mathrm{X})$ & \\
Lid and slit lamp examination & $\mathrm{X}$ & $\mathrm{X}$ & $\mathrm{X}$ & $\mathrm{X}$ & \\
Ophthalmoscopy & $\mathrm{X}$ & & & $\mathrm{X}$ & \\
Intraocular pressure & $\mathrm{X}$ & $\mathrm{X} \mathrm{X} \mathrm{X}$ & $\mathrm{X}$ & $\mathrm{X} \times \mathrm{X}$ & \\
Visual fields & $\mathrm{X}$ & & $\mathrm{X}$ & $\mathrm{X}$ & $\mathrm{X}$ \\
Adverse events & & & $\mathrm{X}$ & $\mathrm{X}$ \\
\hline
\end{tabular}

${ }^{\star}$ Refraction after the baseline examination was only performed if the patient had a loss in visual acuity of two or more lines on the Snellen chart.

At baseline and after 3 months, IOP was determined three times during the day, at $9 \mathrm{am}, 11 \mathrm{am}$, and $5 \mathrm{pm}$.

Table 2 Patient characteristics

\begin{tabular}{lccc}
\hline Characteristics & Latanoprost $(n=112)$ & Dorzolamide $(n=112)$ & Total $(n=224)$ \\
\hline Age (years) & $67(12)$ & $67(10)$ & $67(11)$ \\
Mean (SD) & $29-86$ & $28-94$ & $28-94$ \\
Range & 65 & 65 & 130 \\
Sex: & 47 & 47 & 94 \\
Male & & & 215 \\
Female & 109 & 106 & 2 \\
Race: & 0 & 2 & 6 \\
White & 3 & 3 & 1 \\
Asian & 0 & 1 & 120 \\
Black & 68 & 52 & 5 \\
Other & 2 & & 2 \\
Diagnosis: & 1 & 3 & 88 \\
Primary open angle & 39 & 1 & 9 \\
$\quad$ glaucoma & 2 & 49 & 7 \\
Capsular glaucoma & & & \\
Pigmentary glaucoma & & & \\
Ocular hypertension & Mixed & &
\end{tabular}

$\star$ Patients with different diagnosis in the two eyes.

$\beta$ receptor blockers. After washout the patients were randomised to two parallel study groups: one group received latanoprost $0.005 \%$ once daily in the evening, the other group received dorzolamide $2 \%$ three times daily. The patients were instructed to instil latanoprost at $10 \mathrm{pm}$ and dorzolamide at $9 \mathrm{am}, 2 \mathrm{pm}$, and $10 \mathrm{pm}$.

During the study there were three visits, at baseline, after 2 weeks, and after 3 months of treatment. At baseline and at the 3 month visit IOP was determined at $9 \mathrm{am}, 11 \mathrm{am}$, and $5 \mathrm{pm}$. The 9 am tonometry was performed before the administration of the 9 am dose of dorzolamide in order to ensure optimal timing for subsequent peak and trough measures. The diurnal IOP is defined as the mean value of these three determinations. The IOP was measured with a calibrated Goldmann tonometer. At each time point three separate measurements were taken for each eye and the mean of the three measurements was used in the statistical analysis. Best corrected visual acuity and refraction were determined and a slit lamp examination was performed at all visits. Flare, if present, was graded as mild, moderate, or severe and cells present in a $2 \mathrm{~mm}$ slit were counted and graded as none ( $0-2$ cells), mild ( $3-5$ cells),

Table 3 Reasons for withdrawal from the study

\begin{tabular}{lll}
\hline Reason & $\begin{array}{l}\text { Latanoprost } \\
(n=3)\end{array}$ & $\begin{array}{l}\text { Dorzolamide } \\
(n=5)\end{array}$ \\
\hline Serious adverse event & $1^{\star}$ & $1 \dagger$ \\
Non-compliance to protocol & 2 & 2 \\
Consent withdrawn & 0 & $2 \ddagger$ \\
\hline
\end{tabular}

${ }^{\star}$ Cerebral vascular event.

tColon carcinoma and pulmonary oedema.

¥One patient with facial oedema, one patient with metallic taste and nausea. moderate (6-20 cells), or severe ( $>20$ cells). Any abnormalities of the conjunctiva, cornea, or iris were described and the severity graded as mild, moderate, or severe. A final follow up visit, 2-4 weeks after the end of the study, was scheduled for all patients to follow up any previously noted adverse events or to detect late adverse events. An adverse event was defined as any undesirable event occurring in a subject regardless of whether or not it was considered related to the study drugs. A serious adverse event was defined as an event that was potentially fatal, life threatening, sight threatening, permanently disabling, requiring hospitalisation, or requiring intervention to prevent permanent impairment or damage.

An analysis of covariance (ANCOVA) with diurnal IOP change from baseline to month 3 for the study eye(s) as response variable was carried out. A model was selected from a model based on diurnal IOP on baseline as covariate, treatment group, centre, treatment group by centre, and treatment group by baseline diurnal IOP as factors. From the selected final model the mean difference between latanoprost and dorzolamide in diurnal IOP reduction was estimated and tested. A two sided 95\% confidence interval for the corresponding difference based on the least square estimates from the selected final model was constructed. In addition, the final ANCOVA model was used for analysing the difference between treatments at peak and trough measurements.

\section{Results}

Patient characteristics are presented in Table 2. Of the 224 patients included there were 130 males and 94 females, equally distributed between the two treatment groups. The mean age was 67 years. The most common diagnoses were primary open angle glaucoma $(n=120)$ and ocular hypertension $(n=88)$. The majority of the patients were white. Both eyes were treated in 189 patients and only one eye in 35 patients. There were 74 patients on treatment at the prestudy visit, 36 in the latanoprost group and 38 in the dorzolamide group who had to undergo washout before randomisation. Of these patients 52 were on only one drug before the study, 14 on two drugs and eight on three drugs. The most common drugs used were timolol, betaxolol, levobunolol, dipivefrin, and pilocarpine.

Of the 224 patients enrolled in the study 213 patients were included in the analysis of efficacy. Eight patients were withdrawn, three from the latanoprost group and five from the dorzolamide group. The reasons for withdrawal are shown in Table 3. An additional three patients from the dorzolamide group who completed the study were not included in the efficacy analysis due to major protocol violation. All 224 patients were included in the analysis of safety.

At baseline the mean diurnal IOP was 27.2 (SD 3.0) $\mathrm{mm} \mathrm{Hg}$ in the latanoprost group and 27.2 (3.4) $\mathrm{mm} \mathrm{Hg}$ in the dorzolamide group. At 3 months latanoprost reduced the diurnal IOP by 8.5 (3.3) $\mathrm{mm} \mathrm{Hg}$ compared with 5.6 


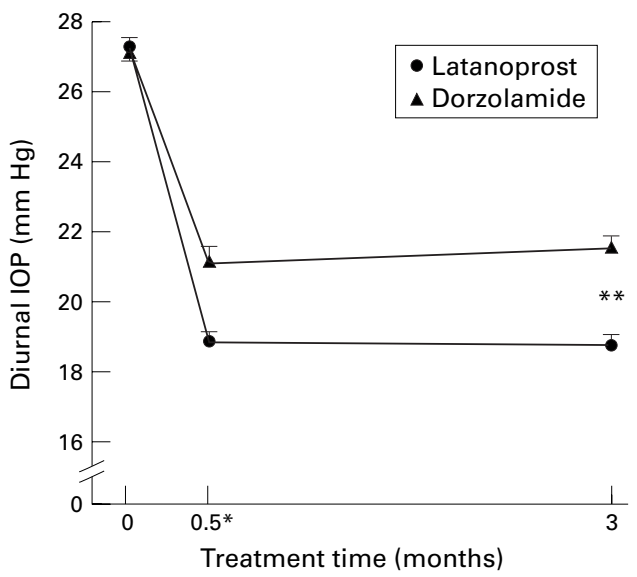

Figure 1 Intraocular pressure (mean (SEM)) at each study visit. ${ }^{\star} I O P$ measured in the morning; ${ }^{\star \star} p<0.001$, latanoprost $v$ dorzolamide.

Table 4 IOP (mm Hg) mean (SD) (n) in each group at each measurement

\begin{tabular}{|c|c|c|}
\hline Time & Latanoprost & Dorzolamide \\
\hline \multicolumn{3}{|l|}{ Baseline } \\
\hline $9 \mathrm{am}$ & $27.7(3.2)(109)$ & $27.8(4.0)(104)$ \\
\hline $11 \mathrm{am}$ & $27.3(3.3)(109)$ & $27.5(3.6)(104)$ \\
\hline $5 \mathrm{pm}$ & $26.5(3.4)(109)$ & $26.4(3.4)(104)$ \\
\hline \multicolumn{3}{|l|}{ Week 2} \\
\hline $11 \mathrm{am}$ & $18.9(3.1)(108)$ & $21.2(3.6)(103)$ \\
\hline \multicolumn{3}{|l|}{ Month 3} \\
\hline $9 \mathrm{am}$ & $18.9(3.4)(109)$ & $23.1(4.0)(104)$ \\
\hline $11 \mathrm{am}$ & $18.7(3.3)(109)$ & $21.3(3.6)(104)$ \\
\hline $5 \mathrm{pm}$ & $18.4(3.1)(109)$ & $20.6(3.4)(104)$ \\
\hline
\end{tabular}

Table 5 The percentage of patients who reached a specific mean diurnal IOP and a specific reduction in mean diurnal IOP at 3 months

\begin{tabular}{lcc}
\hline & $\begin{array}{l}\text { Latanoprost } \\
(n=109)\end{array}$ & $\begin{array}{l}\text { Dorzolamide } \\
(n=104)\end{array}$ \\
\hline IOP $(\mathrm{mm} \mathrm{Hg})$ at 3 months & & \\
$\leqslant 15$ & 9 & 1 \\
$\leqslant 16$ & 17 & 3 \\
$\leqslant 17$ & 28 & 5 \\
$\leqslant 18$ & 46 & 9 \\
$\leqslant 19$ & 60 & 22 \\
$\leqslant 20$ & 72 & 34 \\
$\leqslant 21$ & 82 & 42 \\
Percentage reduction from baseline & \\
$\geqslant 40 \%$ & 21 & 2 \\
$\geqslant 35 \%$ & 37 & 7 \\
$\geqslant 30 \%$ & 52 & 14 \\
$\geqslant 25 \%$ & 72 & 27 \\
$\geqslant 20 \%$ & 87 & 49 \\
$\geqslant 15 \%$ & 93 & 76 \\
\end{tabular}

(2.6) $\mathrm{mm} \mathrm{Hg}$ for dorzolamide (Fig 1). The difference of $2.9 \mathrm{~mm} \mathrm{Hg}$ (95\% CI: 2.3-3.6) was highly significant $(\mathrm{p}<0.001$, ANCOVA $)$. In Table 4 the IOP (mean (SD)) at each time point is shown. The 11 am IOP measurements at baseline and after 3 months can be regarded as representative for the peak effect of the two drugs. Latanoprost reduced IOP at peak by 8.6 $\mathrm{mm} \mathrm{Hg}(32 \%)$ compared with $6.2 \mathrm{~mm} \mathrm{Hg}$ $(23 \%)$ for dorzolamide. Correspondingly, the 5 pm IOP measurements at baseline and after 3 months can be regarded as representative for the trough effect of latanoprost. The 9 am IOP measurements at baseline and after 3 months can be regarded as representative for the trough effect of dorzolamide. Latanoprost reduced IOP at trough by $8.1 \mathrm{~mm} \mathrm{Hg} \mathrm{(31 \% )}$ compared with $4.7 \mathrm{~mm} \mathrm{Hg}(17 \%)$ for dorzolamide. The difference in IOP reduction be- tween treatments at both peak and trough measurements was statistically significant $(p<0.001$, ANCOVA $)$. The percentage of patients who reached a specific mean diurnal IOP and a specific reduction in mean diurnal IOP at 3 months is shown in Table 5. A pressure reduction of at least $30 \%$ was obtained in $52 \%$ of the patients treated with latanoprost compared with $14 \%$ of the patients treated with dorzolamide. A diurnal pressure of $21 \mathrm{~mm} \mathrm{Hg}$ or lower after 3 months of treatment was observed in $82 \%$ of patients treated with latanoprost compared with $42 \%$ of patients on dorzolamide.

There were 173 adverse events reported in 107 patients, 48 on latanoprost, 59 on dorzolamide. Of the 173 adverse events 107 were ocular (50 latanoprost, 57 dorzolamide) and 66 were non-ocular (24 latanoprost and 42 dorzolamide). Ocular adverse events, grouped according to symptoms/signs, are presented in Table 6. Systemic adverse events reported during the study are presented in Table 7 . Most adverse events were graded as mild or moderate.

There were nine serious adverse events in six patients. Two of the serious adverse events resulted in death, a colon carcinoma and pulmonary oedema in an 85 year old man treated with dorzolamide and stroke in an 86 year old man treated with latanoprost. Four patients, two on latanoprost and two on dorzolamide treatment, were hospitalised for planned non-ocular surgery or planned examinations. None of the events was considered to be caused by the study drugs.

Two patients from the dorzolamide group were withdrawn from the study because of non-serious adverse events. In one case the reason was episodes of facial oedema and irritation, and in one case, metallic taste and nausea after eye drop administration.

\section{Discussion}

The present study was undertaken to compare efficacy and side effects of two drugs recently introduced for treatment of open angle glaucoma. The study was open labelled. The two eye drops have different viscosity and different administration regimens which made masking impractical.

Table 6 Ocular adverse events reported in study eyes

\begin{tabular}{lcc}
\hline Adverse event & Latanoprost & Dorzolamide \\
\hline Ocular discomfort & 22 & 29 \\
Eyelid rash/dermatitis & 0 & 3 \\
Hypertrichosis & 1 & 0 \\
Blepharitis & 2 & 0 \\
Epiphora & 0 & 1 \\
Corneal disorder & 2 & 2 \\
Corneal opacity & 1 & 0 \\
Conjunctivitis & 6 & 5 \\
Conjunctival hyperaemia & 1 & 2 \\
Decreased visual acuity & 1 & 0 \\
Blurred vision & 1 & 1 \\
Uncontrolled IOP & 3 & 2 \\
Retinal haemorrhage & 0 & 1 \\
Unspecified eye & 1 & 0 \\
$\quad$ abnormality & & \\
Optic atrophy/pallor & 5 & 7 \\
Deterioration of visual & 4 & \\
$\quad$ field & & 57 \\
Total & 50 &
\end{tabular}


Table 7 Systemic adverse events reported during the study

\begin{tabular}{lcc}
\hline Specification & Latanoprost $^{*}$ & Dorzolamide $^{*}$ \\
\hline Taste perversion & 1 & 12 \\
Respiratory disorders & 3 & 6 \\
Gastrointestinal disorders & 1 & 8 \\
Skin disorders & 2 & 4 \\
Headache/migraine & 3 & 2 \\
Cardiovascular disorders & 2 & 1 \\
Psychiatric disorders & 0 & 2 \\
Endocrine disorders & 1 & 1 \\
Anaemia/nosebleed & 2 & 0 \\
Influenza symptoms & 1 & 1 \\
Prostatic disorder & 1 & 1 \\
Neoplasm & 1 & 1 \\
Other & 6 & 3 \\
Total & 24 & 42 \\
\hline
\end{tabular}

*Latanoprost: congenital haemangioma, ischias, gum and tooth abscess, pain in the face, epileptic aura. Dorzolamide: facial oedema, fatigue, face surgery.

The efficacy of latanoprost has previously been compared with $0.5 \%$ timolol in three 6 months studies..$^{3-5}$ In two of these three studies the IOP reducing effect of latanoprost on diurnal IOP was significantly larger than the effect of timolol. ${ }^{35}$ The effect of dorzolamide on IOP, on the other hand, is less than that of timolol, ${ }^{8}$ which is explained by its weaker aqueous suppressive effect compared with timolol. ${ }^{9}$ Thus, one might expect that latanoprost should be the more effective drug of the two in terms of IOP reduction, an assumption which was confirmed in the present study. The effect of latanoprost on diurnal IOP was almost $3 \mathrm{~mm}$ $\mathrm{Hg}$ more than the effect of dorzolamide, 8.5 $\mathrm{mm} \mathrm{Hg}$ compared with $5.6 \mathrm{~mm} \mathrm{Hg}$. Latanoprost has a long duration of action and is only given once a day. The shorter duration of dorzolamide requires administration three times a day when used as monotherapy. This difference in duration of action is reflected in the effects on IOP at peak and trough. Both latanoprost and dorzolamide can be expected to have a peak effect at $11 \mathrm{am}, 11$ and 2 hours after the previous dose, respectively. The $5 \mathrm{pm}$ measurement should be close to the trough effect for latanoprost, 19 hours after the previous dose, while the 9 am measurement represents the trough effect for dorzolamide, 11 hours after the previous dose. Latanoprost reduced IOP at both peak (11 am) and trough $(5 \mathrm{pm})$ by $31-32 \%$ while dorzolamide reduced IOP at peak by $23 \%(11 \mathrm{am})$ and at trough $(9$ am) by $17 \%$.

Patients with a sustained pressure reduction of $2 \mathrm{~mm} \mathrm{Hg}$ or less were much less frequent in the latanoprost group than in dorzolamide group, $4 \%$ and $15 \%$, respectively. This indicates that the number of non-responders to latanoprost is quite low. The mean untreated pressure was quite high, more than $27 \mathrm{~mm} \mathrm{Hg}$, but a significant majority of the patients, $82 \%$, treated with latanoprost reached an IOP of 21 $\mathrm{mm} \mathrm{Hg}$ or lower after 3 months' treatment, compared with only $42 \%$ of patients on dorzolamide. Less than one third of the patients received glaucoma treatment before the study but previous medications were washed out and no patient had been on any of the study drugs before they entered the study. Thus, the results of the present study demonstrate that the effect on IOP of latanoprost is superior to that of dorzolamide, with regard to peak and trough effects in addition to diurnal IOP effect.

Both study drugs were well tolerated locally and systemically. There were no major differences between the groups. Slightly more adverse events were seen in the dorzolamide group, 99 compared with 74 in the latanoprost group. The difference was mainly the result of more cases with ocular irritation and a metallic taste after eye drop application in patients receiving dorzolamide. No iris pigmentation was reported during the study based on slit lamp examination. Serious adverse events were few and evenly distributed between the two study groups.

In conclusion, latanoprost was superior to dorzolamide in reducing the IOP, judged both from the effect on IOP at peak and trough and by the effect on diurnal IOP. Both drugs were well tolerated during the study period.

Supported by a grant from Pharmacia and Upjohn, United Kingdom.

\section{Appendix}

Members of the UK and Ireland Latanoprost Study Group: Coordinating investigator: Eamonn O'Donoghue, Galway, Ireland.

Principal investigators: Mike Austin, Singleton Hospital, Swansea; Keith Bates, Taunton and Somerset Hospital, Taunton; Mike Birch, Royal Victoria Hospital, Newcastle; Louis Clearkin, Arrow Park Hospital, Merseyside; Patrick Condon, Waterford Regional Hospital, Ireland; Bruce James, Stoke Mandeville Hospital, Aylesbury; David O'Brart, St Thomas's Hospital, London; Peter Phelan, Sunderland Eye Infirmary Hospital, Sunderland; Rosemary Robinson, Coventry and Warwickshire Hospital, Coventry; Sanjay Shah, St Bartholomew's Hospital, London; Fiona Spencer, Royal Eye and Ear Hospital, Manchester.

1 Wilkerson M, Cyrlin M, Lippa EA, et al. Four-week safety and efficacy study of dorzolamide, a novel, active topical carbonic anhydrase inhibitor. Arch Ophthalmol 1993;111:1343-50.

2 Maus TL, Larsson L-I, McLaren JW, et al. Comparison of dorzolamide and acetazolamide as suppressors of aqueous humor flow in humans. Arch Ophthalmol 1997;115:45-9.

3 Alm A, Stjernschantz J, the Scandinavian Latanoprost Alm A, Stjernschantz J, the Scandinavian Latanoprost
Study Group. Effects on intraocular pressure and side effects of $0.005 \%$ latanoprost applied once daily, evening or morning. A comparison with timolol. Ophthalmology 1995; 102:1743-52.

102:1743-52.
Watson P, Stjernschantz J, the Latanoprost Study Group. A Watson P, Stjernschantz J, the Latanoprost Study Group. A
six-month, randomized, double-masked study comparing latanoprost with timolol in open-angle glaucoma and ocular hypertension. Ophthalmology 1996;103:126-37.

5 Camras CB, the United States Latanoprost Study Group. Comparison of latanoprost and timolol in patients with ocular hypertension and glaucoma: a six-month, masked, multicenter trial in the United States. Ophthalmology 1996; 103:138-47.

6 Stjernschantz J, Selén G, Sjöquist B, et al. Preclinical pharmacology of latanoprost, a phenyl-substituted PGF analogue. Adv Prostaglandin Thromboxane Leukot Res 1995; 23:513-8.

7 Toris CB, Camras CB, Yablonski ME. Effects of PhXA41, a new prostaglandin $\mathrm{F}_{2 \alpha}$ analog, on aqueous humor dynamics in human eyes. Ophthalmology 1993;100:1297-304.

8 Strahlman E, Tipping R, Vogel R, et al. A double-masked, randomized 1-year study comparing dorzolamide (Trurandomized 1-year study comparing dorzolamide (Trusopt), timo

9 Wayman L, Larsson L-I, Maus T, et al. Comparison of dorzolamide and timolol as suppressors of aqueous humor flow in humans. Arch Ophthalmol 1997;115:1368-71. 